\title{
The Effect of Foam Rolling on Recovery Between Two Eight Hundred Metre Runs
}

\author{
by \\ Anthony $D^{\prime}$ Amico $^{1}$, Vincent Paolone ${ }^{2}$
}

With the increased popularity of foam rolling as a means of recovery, it is important to establish the exact manner in which the practice is useful. The purpose of this study was to examine the impact of foam rolling on recovery between two $800 \mathrm{~m}$ runs. Sixteen trained males (mean \pm sd; age, $20.5 \pm .5 \mathrm{yr}$; average $800 \mathrm{~m}$ treadmill run time, 145.2 $\pm 1.8 \mathrm{~s})$ participated in the study, using a randomized, crossover design. The subjects completed two $800 \mathrm{~m}$ runs on a treadmill, separated by a $30 \mathrm{~min}$ rest, during which time a foam rolling protocol or passive rest period was performed. The speed of each run was as fast as possible. Subjects had access to speed controls, but were blinded to the actual speed. Blood lactate concentration and VCO2 were measured prior to and following each run. Stride length, $800 \mathrm{~m}$ run time, and hip extension were measured during each run. VCO2, stride length, $800 \mathrm{~m}$ run time, and hip extension were not significantly different between conditions $(p>.05)$. For blood lactate, no statistical interaction was found between condition and time $(p>.05)$. Foam rolling between two $800 \mathrm{~m}$ runs separated by 30 min performed by trained male runners does not alter performance.

Key words: lactate, range of motion, foam rolling.

\section{Introduction}

Recovery between physiologically stressful exercise bouts is paramount to athletic success (Barnett, 2006). Complete recovery is not always possible due to the repetitive nature of many athletic competitions, so utilizing the available time as effectively as possible can provide a major competitive advantage (Barnett, 2006; Bielik, 2010; Stanula et al., 2014). Massage is often utilized to enhance the recovery process (Weerapong et al., 2005), but the practicality of massage is limited in many athletic settings. Foam rolling (FR) may offer comparable benefits to massage, but with far greater accessibility.

FR involves the movement of varying limbs over cylindrical, foam objects. While limited evidence suggests that the practice does not acutely improve athletic performance despite lowering post-exercise fatigue (Healey et al., 2014), FR has been found to increase joint range of motion (ROM) while preserving strength and power (Macdonald et al., 2013). Researchers have also suggested that FR may reduce the sensation of delayed onset muscle soreness (DOMS) (Macdonald et al., 2014), and could expedite the restoration of athletic performance following fatiguing exercise over a multiple-day span (Macdonald et al., 2014; Pearcey et al., 2015). To our knowledge, there are no studies in the literature examining the influence of $\mathrm{FR}$ on recovery between exercise bouts spaced by less than 24 hours.

The successful clearance of lactate is a

1 - Salem State University 352 Lafayette Street, Salem, MA, USA.

2- Springfield College, 263 Alden Street, Springfield, MA, USA. 
commonly utilized recovery marker between two closely-spaced bouts of primarily glycolytic exercise, such as a maximal $800 \mathrm{~m}$ run (Astrand et al., 1986; Bret et al., 2003; Dolgener and Morien, 1993; Donovan and Pagliassotti, 2000). Lactate production that exceeds lactate clearance is generally associated with highly intense bouts of exercise. While rising blood lactate levels indeed indicate that ATP is being utilized faster than it can be resynthesized aerobically and that exhaustion may be imminent, lactate itself is not the driving force behind the decline in performance (Lamb and Stephenson, 2006). Muscular fatigue occurs due to a broad array of ionic and metabolic factors that can interfere with excitation-contraction coupling during exercise, but the direct influence of blood lactate does not appear to be one of them (Lamb and Stephenson, 2006). Nevertheless, even if accelerated production of lactate does not contribute to fatigue, an accumulation of lactate in the blood is reflective of an unsustainable intensity of exercise (Lamb and Stephenson, 2006). Interventions that attenuate measured blood lactate levels may reflect the potential for a higher exercise intensity to be utilized, or for a given intensity to be sustained longer. In an event such as the $800 \mathrm{~m}$ run, both of these outcomes could enhance performance. While the influence of FR on lactate clearance has not been investigated, light exercise or active recovery between subsequent exercise bouts appears to enhance removal and improve performance compared to passive rest (Ali Rasooli et al., 2012; Barnett, 2006; Bielik, 2010).

Given the varied purported benefits of $\mathrm{FR}$, any influence on recovery and performance could be attributed to a handful of possible mechanisms. FR is generally executed in an active, rhythmical fashion (MacDonald et al., 2013). Thus, it is reasonable to speculate that the practice could influence lactate clearance in a similar manner to active recovery and improve subsequent exercise bout performance (Ali Rasooli et al., 2012; Barnett, 2006; Bielik, 2010). Furthermore, given the increase in ROM associated with FR (Bushell et al., 2015; Feldbauer et al., 2015; Macdonald et al., 2013; Mohr et al., 2014; Sullivan et al., 2013), it is plausible that the practice could favorably influence dynamic hip extension ROM and stride length while running. Therefore, the purpose of our study was to determine the effects of foam rolling on recovery between two $800 \mathrm{~m}$ runs separated by $30 \mathrm{~min}$. We specifically addressed the effects of FR on $800 \mathrm{~m}$ run time, blood lactate, $\mathrm{VCO}_{2}$, hip extension $\mathrm{ROM}$, and stride length.

\section{Material and Methods}

\section{Participants}

Sixteen trained males (age $20.5 \pm 3.3 \mathrm{yr}$, body height $174.7 \pm 6.6 \mathrm{~cm}$, body mass $70.8 \pm 7.9$ $\mathrm{kg}$, body fat $10.1 \pm 4.1 \%$, average $800 \mathrm{~m}$ treadmill run time $145.2 \pm 1.8 \mathrm{~s}$ ) volunteered for and gave written informed consent to participate in the current study, which was approved by the Springfield College Institutional Review Board. Individuals who were not trained to perform and recover from intense, glycolytic running were not included. Subjects were required to demonstrate the ability to run $800 \mathrm{~m}$ on a treadmill in less than $160 \mathrm{~s}$ prior to participation. Two potential subjects were not able to successfully complete the screening test and were excluded from the study. The reported average $800 \mathrm{~m}$ treadmill run time was the mean time to complete the initial run of each testing session. The subjects were instructed to refrain from vigorous exercise for 24 hours prior to each testing session, and were told to abstain from caffeine and alcohol for 12 hours prior to each testing session. The subjects also kept a dietary record for three days prior to each testing session and were told to maintain a similar diet prior to each test.

Procedures

We used a repeated-measures design to examine the effect of foam rolling on $800 \mathrm{~m}$ run performance, blood lactate concentration, $\mathrm{VCO}_{2}$, hip extension ROM, and stride length. All subjects participated in one familiarization session and two testing sessions. The two testing sessions were comprised of an experimental session when foam rolling was performed, and a control session utilizing passive rest. The order of these sessions was randomized, with half of the subjects foam rolling during their first testing session, and half in the second testing session. Testing sessions were separated by at least 72 hours. Testing occurred at the same time for each testing session to control for diurnal variation and circadian rhythms.

During the first familiarization session, subjects were provided with a verbal explanation of the study, and read and signed an informed 
consent form. Subjects also completed a medical history questionnaire. Subject's age, body height, body mass, and body fat percentage were recorded. The body fat percentage was estimated via air displacement plethysmography using a Bod Pod (Cosmed, Rome, Italy). All running was performed on a motorized treadmill (PulsarPnewTrack 28AX treadmill $(\mathrm{h} / \mathrm{p} / \mathrm{cosmos}$, Traunstein, Germany). The sessions were performed with subject's respiratory gases collected by a PhysioDyne Max-II open circuit spirometry system (AEI Technologies, Pittsburgh, PA). After a $5 \mathrm{~min}$ warm-up at a self-selected pace, treadmill speed was set at $18 \mathrm{~km} / \mathrm{h}$. If subjects maintained that pace for $160 \mathrm{~s}$, they were eligible to participate in the study.

Following successful completion of this exercise test, subjects were introduced to the FR protocol on a Premium EVA 12" X 6", Round roller (Power Systems, Nashville, TN) that was utilized in the study. Subjects were instructed to place their body weight on the roller, and the gluteal muscles, hip flexors, quadriceps, iliotibial (IT) bands, adductors, and calves were rolled in a rhythmical pattern, utilizing 6 slow passes over each area totaling $30 \mathrm{~s}$. Each pass in either direction lasted $5 \mathrm{~s}$. Each roll began at the respective origin of the targeted muscle, with subjects instructed to return in the opposite direction once reaching the insertion. Once the foam rolling familiarization was complete, subjects were randomly divided into groups that foam rolled during the first or second testing session.

During testing sessions, subjects completed two $800 \mathrm{~m}$ treadmill runs, separated by $30 \mathrm{~min}$ of passive recovery between bouts. Upon entering the lab, subjects were affixed with white masking tape at the left greater trochanter and the lateral epicondyle of the knee to serve as markers for motion analysis. Subjects were provided with water that could be consumed ad libitum between $800 \mathrm{~m}$ bouts. The amount consumed in the first testing session was matched during the second testing session. Subjects were equipped with the head mask, mouthpiece, and ventilator hose associated with the open circuit spirometry system, and performed a 5 min warm-up on the treadmill at a self-selected pace. At the conclusion of the warm-up, capillary blood was drawn from the fingertips of the subjects to determine blood lactate concentration using an Accusport lactate analyzer (Sports Resource Group, Hawthorne, NY). Blood samples were taken immediately prior to and following each $800 \mathrm{~m}$ bout. The intensity of the two runs was self-selected, with subjects encouraged to run as fast as possible. Subjects had access to the speed controls, but were blinded to the actual speed. Each run was initiated at a treadmill speed of $20.6 \mathrm{~km} / \mathrm{h}$. Subjects then signaled over the course of the run whether to increase or decrease treadmill speed. Run time was calculated as the time to complete the $800 \mathrm{~m}$ distance.

Upon completion of the first run, capillary blood was again drawn, and open circuit spirometry equipment was removed. Subjects in both conditions then sat quietly for $15 \mathrm{~min}$. In the experimental condition, subjects then spent 10 min performing the aforementioned FR protocol, then returned to the treadmill and awaited the beginning of their second exercise bout. In the control condition, subjects spent $25 \mathrm{~min}$ sitting quietly, then returned to the treadmill and awaited the beginning of their second bout while open circuit spirometry equipment was re-affixed. After $30 \mathrm{~min}$ had passed since the conclusion of the first run, subjects in both conditions performed a second $5 \mathrm{~min}$ warm-up at a selfselected pace. After a blood sample was taken, subjects performed a second maximal $800 \mathrm{~m}$ run, conducted in the same fashion as the first.

All $800 \mathrm{~m}$ run bouts were visually recorded with a MacBook Pro FaceTime HD camera, shot at 30 FPS (Apple Inc., Cupertino, CA). Video recordings were shot from a stationary, sagittal vantage point capturing each subject's entire body. Recordings were initiated immediately prior to each $800 \mathrm{~m}$ run, and terminated shortly after the conclusion. Hip extension was assessed by measuring the angle between the midline of the torso, and a line between the greater trochanter and lateral epicondyle of the knee. A measurement was recorded at maximal extension during the run stride at the 30,60, and $90 \mathrm{~s}$ mark of each run. An average was calculated for each run. Analysis was completed using Dartfish software (Dartfish USA, Inc., Alpharetta, GA). Analysis was not performed by a blinded technician. Stride length was operationally defined as the distance between the subject's left toe at the initial strike and toe off phase, and 
calculated based on the formula described by Millet et al. (2000).

\section{Statistical Analysis}

Two $2 \times 4$ Analysis of Variance (ANOVA) with two repeated measures factors, along with three $2 \times 2$ repeated measures ANOVAs were used to analyze the data. Blood lactate and $\mathrm{VCO}_{2}$ were measured with the $2 \times 4$ RM ANOVAs. 800 $\mathrm{m}$ run time, hip extension ROM, and stride length were measured with the $2 \times 2$ RM ANOVAs. For the $2 \times 4$ ANOVA, the first repeated measures factor was condition. Foam rolling served as the experimental condition, while passive rest served as the control. The second repeated measures factor was time, with the four levels being a pre initial $800 \mathrm{~m}$ run, post initial $800 \mathrm{~m}$ run, pre subsequent $800 \mathrm{~m}$ run, and post subsequent $800 \mathrm{~m}$ run. Dependent variables were $800 \mathrm{~m}$ run time and blood lactate. The Mauchly's test of sphericity was used to assess homogeneity of variance. If this assumption was violated, then the Greenhouse-Geisser adjustment was utilized. To assess practical significance, magnitude-based inferences were determined using eta squared $\left(\eta^{2}\right)$. Effect size was interpreted using the following criteria: small, $>.02$, medium, $>.13$, large, $>$.26. IBM SPSS v. 20 was used to analyze all data. Alpha level was set at $p=.05$.

\section{Results}

Figure 2 depicts the mean \pm SD of stride length, $800 \mathrm{~m}$ run time, and hip extension. Figure 3 depicts the mean \pm SD of blood lactate and $\mathrm{VCO}_{2}$.

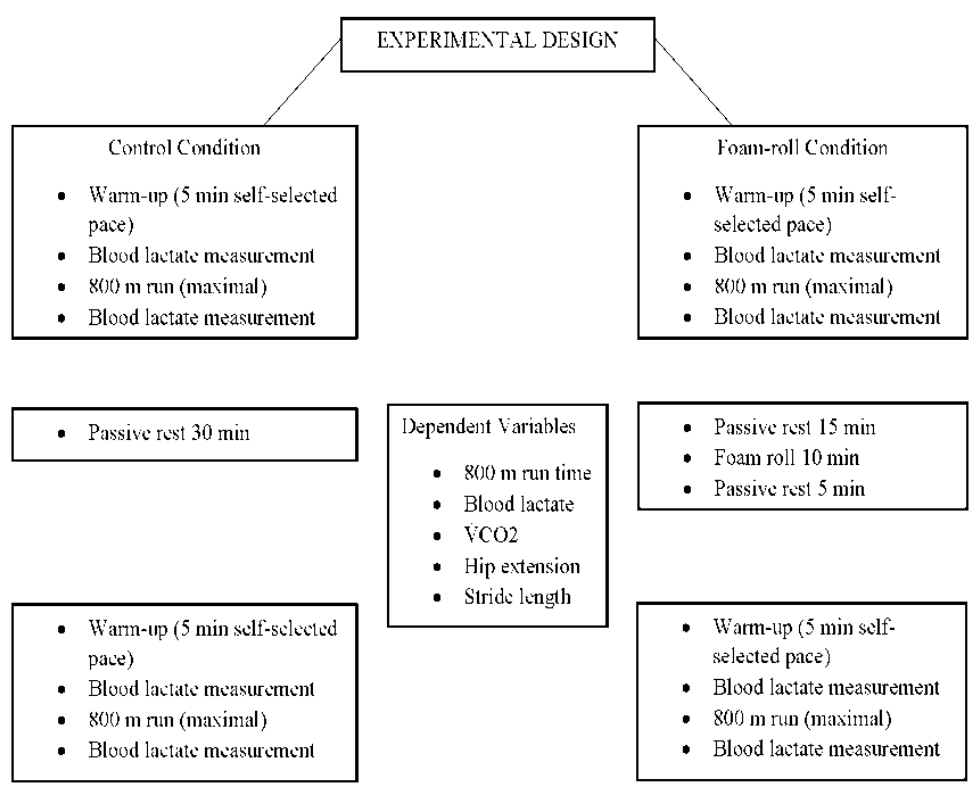

Figure 1

Methodology. Flow chart displays methodology, along with the dependent variables assessed during each test 
A

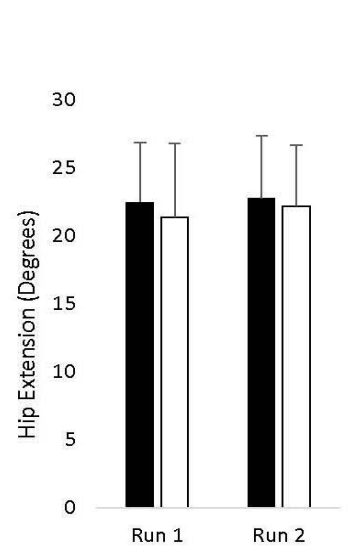

B

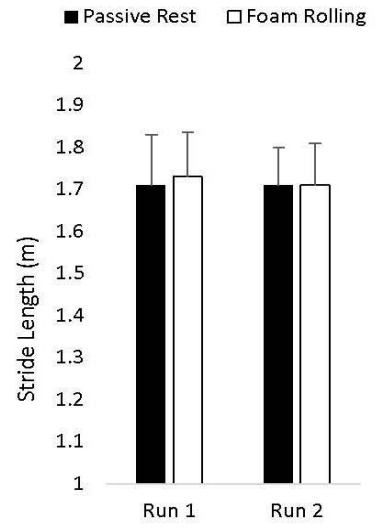

C

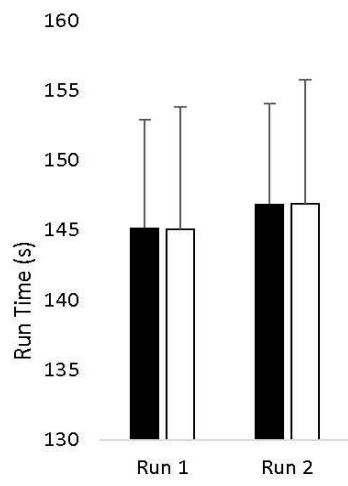

Figure 2

(A) Hip Extension, (B) Stride Length, (C) Run Time values during the first and second $800 \mathrm{~m}$ run, in the passive rest and foam rolling conditions

A

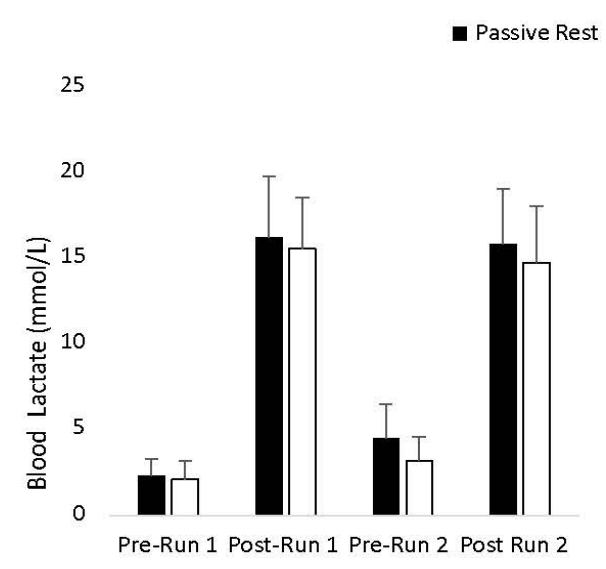

B

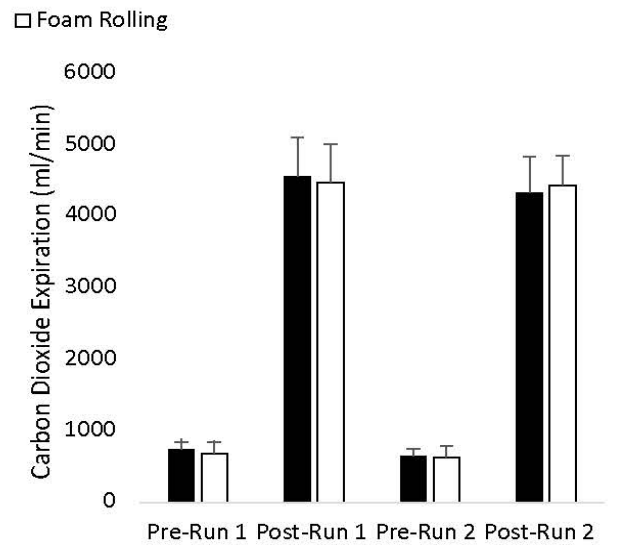

Figure 3

(A) Blood Lactate, (B) Carbon Dioxide Expiration values prior to and following the first and second $800 \mathrm{~m}$ run, in the passive rest and foam 
No significant interactions $(p>.05)$ were found among any variables. A small effect size $\left(\eta^{2}=.07\right)$ was observed for the blood lactate time and condition interaction. In the control condition, blood lactate was $2.3 \pm 1.0 \mathrm{mmol} / \mathrm{L}$ at pre-run 1 , $16.2 \pm 3.5 \mathrm{mmol} / \mathrm{L}$ at post-run $1,4.5 \pm 2.0 \mathrm{mmol} / \mathrm{L}$ pre-run 2 , and $15.8 \pm 3.2 \mathrm{mmol} / \mathrm{L}$ post-run 2 . In the foam rolling condition, blood lactate was $2.1 \pm 1.1$ $\mathrm{mmol} / \mathrm{L}$ at pre-run $1,15.5 \pm 3.0 \mathrm{mmol} / \mathrm{L}$ at postrun $1,3.2 \pm 1.4 \mathrm{mmol} / \mathrm{L}$ at pre-run 2 , and $14.7 \pm 3.3$ $\mathrm{mmol} / \mathrm{L}$ at post-run 2. For time, the following variables were found to be significant $(p<.05)$ : blood lactate concentration $\left(p=.00, \eta^{2}=.96\right)$, and run time $\left(p=.01, \eta^{2}=.39\right)$. The post hoc analysis for blood lactate concentration revealed expected physiological responses to exercise. Run time was significantly lower in time $1(145.2 \pm 1.8 \mathrm{~s})$ compared to time $2(146.9 \pm 2.2 \mathrm{~s})$. For condition, no significant differences $\left(p=.92, \eta^{2}=.00\right)$ were found for run time.

\section{Discussion}

The main finding of the present investigation was that FR between two $800 \mathrm{~m}$ runs separated by $30 \mathrm{~min}$ did not improve recovery or performance. To our knowledge, this is the first study to investigate the influence of FR on shortterm exercise recovery. A small number of investigations have been performed using longer recovery periods. Macdonald et al. (2014) showed that FR can reduce DOMS and attenuate the associated decrements in performance. Subjects performed lower body FR exercises immediately afterwards and in the days following a highvolume squat protocol. DOMS was significantly reduced at 24, 48, and 72 hours post-exercise compared to a non-FR control (Macdonald et al., 2014). Vertical jump (VJ) results were also maintained whereas the control group experienced reductions in the VJ. Pearcey et al. (2015) carried out a similar investigation using the same high-volume squat protocol. Those authors reported that FR reduced DOMS, increased the pressure-pain threshold score, and reduced decrements in speed, power, and dynamic strength-endurance. Taken together, the results of these studies appear to indicate that FR is an effective method for reducing DOMS and enhancing recovery following challenging resistance exercise (Macdonald et al., 2014; Pearcey et al., 2015). The results of the present investigation appear to suggest that FR may not influence short-term recovery in the same beneficial manner. Also, FR may not influence recovery from running in the same manner as recovery from resistance training. One notable difference between the present investigation and those previously mentioned is the duration of the FR protocol. Both of the aforementioned studies used foam rolling protocols $20 \mathrm{~min}$ in length. The present investigation used a shorter, $10 \mathrm{~min}$ protocol intended to mimic those most commonly observed in the field. Future investigations should examine whether or not longer FR protocols benefit short-term recovery from running in the same manner as longer-term recovery from resistance exercise.

In the present investigation, blood lactate and $\mathrm{VCO}_{2}$ were not lower in the experimental condition, indicating that FR may not influence $\mathrm{pH}$ between two $800 \mathrm{~m}$ runs. Martin et al. (1998) reported that blood lactate clearance was not enhanced with sports massage. Reporting similar findings, Bielik (2010) stated that massage did not reduce blood lactate more effectively than passive rest, and further reported that active recovery, i.e.; submaximal cycling, was a more effective practice for reducing blood lactate between exercise bouts. The results of the present investigation suggest that foam rolling, similar to massage, may not influence lactate turnover despite its active nature.

Micklewright et al. (2006) also reported that lactate measurements were not reduced with massage, but a combination of massage and active recovery resulted in increased lactate clearance compared to passive rest, and equivalent lactate clearance compared to active recovery alone. Given that the combined massage and active recovery group performed half the exercise as the active recovery-only group, the authors suggested that the combined protocol was a more economical one (Micklewright et al., 2006). Those authors also suggested that the massage portion of combined massage-active recovery may enhance lactate removal via an increase in intracellular hydrostatic pressure. The increase in hydrostatic pressure may enhance diffusion across the sarcolemma, and increase the rate at which lactate exits the intracellular space and enters the extracellular space. Micklewright et al. (2006) further stated that this process would allow 
for more lactate to be utilized via hepatic gluconeogenesis. Active recovery, in contrast, may enhance lactate clearance via an increase in oxidation by the utilized musculature (Micklewright et al., 2006). During active recovery, the continually exercising muscles enhance lactate uptake, and the low exercise intensity maintains a favorable gradient for this uptake (Micklewright et al., 2006). In isolation, massage does not appear to increase lactate turnover (Micklewright et al., 2006). Active recovery independent of any other treatment does appear to increase lactate turnover (Micklewright et al., 2006). Combining the two techniques may enhance lactate turnover in a more economical fashion than active recovery alone (Micklewright et al., 2006). Results from the present investigation indicate that FR may not provide adequate external pressure to the tissues to enhance outward lactate diffusion. FR also may not impart a sufficient metabolic demand to replicate traditional active rest, such as light cycling. Though a small effect size $(\eta 2=.07)$ was found for the interaction, statistical significance was not reached. While this could be interpreted as a trend towards meaningfully lower lactate levels in the FR condition, it should be noted that average blood lactate levels were lower before the treatment had even occurred following the first $800 \mathrm{~m}$ run. This may indicate that the observed outcomes in blood lactate were due to random chance, as opposed to the FR intervention. Further research is needed to clarify this relationship. In light of the aforementioned findings, those seeking to clear lactate as effectively as possible between exercise bouts should utilize active recovery methods, and may consider combined active recovery and massage (Micklewright et al., 2006).

In the present investigation, run time did not differ between FR and passive rest conditions. The findings of the current study appear to contradict those of Ali Rasooli et al. (2012) who reported that while massage did not improve lactate clearance compared to passive recovery, it did improve swim times in the $200 \mathrm{~m}$ front crawl. Those authors were not able to attribute these findings to any specific physiological occurrence, and the improved performance of the swimmers was thought to be due to some type of psychological benefit. Ali Rasooli et al. (2012) speculated that massage may confer an enhanced sense of well-being, which may contribute to improved performance by an athlete. Healey et al. (2014) reported that while foam rolling resulted in lower levels of post-exercise fatigue, there was no effect on performance in a series of athletic tests assessing jump height and power, isometric force, and agility. In the present investigation, the lack of improvement in performance was consistent with that reported by Healey et al. (2014). While massage performed during a short recovery period has been shown to improve performance (Ali Rasooli et al., 2012), FR has not been demonstrated to offer the same benefit. A potential explanation for this contrast may be the purported psychological benefit derived from massage treatment (Ali Rasooli et al., 2012). A treatment applied by the hands of a skilled practitioner may not influence the recipient in the same manner as FR.

FR did not influence hip extension ROM or stride length measured during the run tests. Macdonald et al. (2013) reported that a FR protocol transiently increased joint ROM without a concurrent decrease in muscular force production. It does not appear that these benefits extend to $800 \mathrm{~m}$ run performance, or potentially measures of dynamic ROM. A possible explanation is that the FR protocol utilized in the investigation was applied to a variety of lower body locations. The gluteal muscles, hip flexors, quadriceps, (IT) bands, adductors, and calves were rolled during the protocol, but only hip extension was measured via video analysis. The total time spent on the areas that must be treated to optimize hip extension may have been insufficient in the current study. Sullivan et al. (2013) noted a dose-response effect, reporting that longer duration rolling bouts were more effective than shorter bouts for increasing joint ROM as measured by a goniometer. MacDonald et al. (2013) reported that two 1 min rolling bouts of the quadriceps increased knee ROM as measured by a goniometer. The objective of the current study was to assess the most commonly utilized FR protocols in the field, and replicate the manufacturer's instructions. The utilized protocol may not have allotted sufficient time to each muscle group to elicit changes in joint ROM. Furthermore, the increases in static joint ROM reported by MacDonald et al. (2013) may not 
necessarily carry over to dynamic joint ROM during a running stride.

The present study specifically investigated the influence of $\mathrm{FR}$ on middle distance running. The findings may be limited to that activity or individuals conditioned for that intensity and duration of exercise. Another limitation is that the FR protocol used in the present study took approximately $10 \mathrm{~min}$ to complete. FR bouts of different durations may alter outcomes. The findings of this investigation may be limited to middle distance running on a treadmill as well. While subjects were provided with a familiarization session, running on a treadmill instead of a track may have exerted excessive influence on performance outcomes. Additionally, subjects may have adhered too closely to the arbitrary $20.6 \mathrm{~km} / \mathrm{h}$ starting speed on the treadmill, despite instructions from the investigators to complete the run as quickly as possible. Furthermore, an inherent limitation of research involving any type of soft tissue treatment is the inability to blind subjects. Future researchers may consider utilizing sham treatments in an effort to control for possible placebo effects.

\section{Conclusion}

The lack of difference between FR and passive rest suggest that the former may not be an effective means of recovery between highintensity exercise bouts separated by $30 \mathrm{~min}$. FR should continue to be utilized as a means to recover from resistance training-induced DOMS, and as a method of increasing joint ROM while maintaining strength and power. Light exercise as a means of active recovery is likely a superior method to FR for minimizing fatigue-induced decrements in performance during successive exercise bouts. Future researchers should continue to examine optimal ways for athletes who compete multiple times per day in very high intensity events to recover between bouts. Additionally, future researchers should further investigate the effects of FR on exercise bouts separated by greater amounts of time, and the extent to which FR might influence DOMS. Maximizing recovery between bouts or competitions is critical to the success of any athlete. Identifying exactly how and when FR is effective, and when it is not, will help athletes and coaches make well-informed decisions regarding the training and recovery methods that they choose to employ.

\section{Acknowledgements}

The results of this investigation were presented in the abstract form at the New England ACSM Regional Meeting, October 13, 2015, and the ACSM Annual Meeting, June 2, 2016. No financial support was received for this study. Thank you to Dr. Tracey Matthews of Springfield College for assisting with the statistical analysis used in this investigation. Thank you to Dr. Richard Wood of Springfield College for lending technical expertise and assisting with the study design. Thank you to the athletes and coaches who volunteered to participate.

Anthony D'Amico was the principal investigator of this study, and Vincent Paolone supervised and contributed extensively to the design.

\section{References}

Ali Rasooli S, Koushkie Jahromi M, Asadmanesh A, Salesi M. Influence of massage, active and passive recovery on swimming performance and blood lactate. J Sports Med Phys Fitness, 2012; 52: 122-127

Astrand P, Hultman E, Juhlin-Dannfelt A, Reynolds G. Disposal of lactate during and after strenuous exercise in humans. J Appl Phys, 1986; 61: 338-343

Barnett A. Using recovery modalities between training sessions in elite athletes: Does it help? Sports Med, 2006; 36: 781-796

Bielik VV. Effect of different recovery modalities on anaerobic power in off-road cyclists. Bio Sport, 2010; 27: 59-63

Bret C, Messonnier L, Nouck Nouck J, Freund H, Dufour A, Lacour J. Differences in lactate exchange and 
removal abilities in athletes specialised in different track running events (100 to $1500 \mathrm{~m})$. Int J Sports Med, 2003; 24: 108-113

Bushell JE, Dawson SM, Webster MM. Clinical relevance of foam rolling on hip extension angle in a functional lunge position. J Strength Cond Res, 2015; 29: 2397-403

Dolgener FA, Morien AA. The effect of massage on lactate disappearance. J Strength Cond Res, 1993; 7: 159162

Donovan CM, Pagliassotti MJ. Quantitative assessment of pathways for lactate disposal in skeletal muscle fiber types. Med Sci Sports Exerc, 2000; 32: 772-777

Feldbauer CM, Smith BA, Van Lunen B. The effects of self-myofascial release on flexibility of the lower extremity: a critically appraised topic. Int J Athl Ther Train, 2015; 20: 14-19

Healey KC, Hatfield DL, Blanpied P, Dorfman LR, Riebe, D. The effects of myofascial release with foam rolling on performance. J Strength Cond Res, 2014; 28: 61-68

Lamb GD, Stephenson DG. Point: lactic acid accumulation is an advantage during muscle activity. J Appl Physiol, 2006; 100: 1410-2; discussion 1414

Macdonald GZ, Button DC, Drinkwater EJ, Behm DG. Foam rolling as a recovery tool after an intense bout of physical activity. Med Sci Sports Exerc, 2014; 46: 131-42

Macdonald GZ, Penney M, Mullaley ME, Cuconato AL, Drake CD, Behm DG, Button DC. An acute bout of self-myofascial release increases range of motion without a subsequent decrease in muscle activation or force. J Strength Cond Res, 2013; 27: 812-821

Martin NA, Zoeller RF, Robertson RJ, Lephart SM. The comparative effects of sports massage, active recovery, and rest in promoting blood lactate clearance after supramaximal leg exercise. J Athl Train, 1998; 33: 30-35

Millet GP, Millet GY, Hofmann MD, Candu RB. Alterations in running economy and mechanics after maximal cycling in triathletes: influence of performance level. Int J Sports Med, 2000; 21: 127-32

Micklewright DD, Sellens MM, Gladwell VV, Beneke RR. Blood lactate removal using combined massage and active recovery. Bio Sport, 2006; 23: 315-325

Mohr A, Long B, Goad C. Foam rolling and static stretching on passive hip flexion range of motion. J Sport Rehabil, 2014; 23: 296-9

Pearcey GP, Bradbury-Squires DJ, Kawamoto J, Drinkwater EJ, Behm DG, Button DC. Foam rolling for delayed-onset muscle soreness and recovery of dynamic performance measures. J Athl Train, 2015; 50: 5-13

Stanula A, Roczniok R, Maszczyk A, Pietraszewski P, ZajacA. The role of aerobic capacity in high-intensity intermittent efforts on ice-hockey. J Hum Kinet, 2014; 31(3): 193-199

Sullivan KM, Silvey DB, Button DC, Behm DG. Roller-massager application to the hamstrings increases sitand-reach range of motion within five to ten seconds without performance impairments. Int J Sports Phys Ther, 2013; 8: 228-236

Weerapong P, Hume PA, Kolt GS. The mechanisms of massage and effects on performance, muscle recovery and injury prevention. Sports Med, 2005; 35: 235-256

\section{Corresponding author:}

\section{Anthony D'Amico}

Salem State University

352 Lafayette Street, Salem, MA, USA 01970

Fax: 978-542-6554

Tel: 978-542-2904

E-mail: adamico@salemstate.edu 